\title{
Assessment of Cardiac Autonomic Nervous System Involvement in Systemic Sclerosis via Exercise Heart Rate Recovery
}

\author{
Ugur Nadir Karakulak ${ }^{a}$ Sercan Okutucu ${ }^{a}$ Levent Şahiner ${ }^{a} \quad$ Naresh Maharjan $^{a}$ \\ Elifcan Aladag ${ }^{b} \quad$ Ali Akdogan $^{b} \quad$ Levent Kilic $^{\mathrm{b}}$ Ergun Baris Kaya ${ }^{a}$ \\ Kudret Aytemir ${ }^{a} \quad$ Lale Tokgozoglu ${ }^{a}$ \\ Departments of a Cardiology and ${ }^{b}$ Internal Medicine, Division of Rheumatology, Faculty of Medicine, Hacettepe \\ University, Ankara, Turkey
}

\section{Key Words}

Cardiac autonomic dysfunction - Heart rate recovery .

Scleroderma

\section{Abstract}

Objective: To assess exercise heart rate recovery (HRR) indices in patients with systemic sclerosis (SSc) for an assessment of their cardiac autonomic function. Subjects and Methods: Thirty-five patients with diffuse or limited SSc and 35 healthy controls were enrolled. All subjects underwent exercise testing and transthoracic echocardiography. The HRR indices were calculated by subtracting the first- (HRR1), second- (HRR2) and third-minute (HRR3) heart rates from the maximal heart rate. Results: The SSC and control groups were similar in age ( $45.2 \pm 11.6$ vs. $43.9 \pm 10.0$ years), had identical gender ratios (31 female/4 male in both groups) and similar left ventricular ejection fraction (66.5 \pm 5.1 vs. $67.7 \pm 5.9 \%)$. The mean HRR1 (21.8 \pm 4.4 vs. $27.7 \pm 4.3 \mathrm{bpm}$, $\mathrm{p}=0.001), \operatorname{HRR} 2(43.8 \pm 6.3 \mathrm{vs} .47 .6 \pm 4.4 \mathrm{bpm}, \mathrm{p}=0.004)$ and HRR3 (58.8 \pm 10.3 vs. $63.6 \pm 7.3 \mathrm{bpm}, \mathrm{p}=0.031)$ values were significantly lower in the SSc group than in the healthy con-

\begin{tabular}{ll}
\hline KARGER 125/\% & $\begin{array}{l}\text { @ 2014 S. Karger AG, Basel } \\
\text { 1011-7571/14/0241-0017\$39.50/0 Openger }\end{array}$ \\
$\begin{array}{l}\text { E-Mail karger@karger.com } \\
\text { www.karger.com/mpp }\end{array}$ & $\begin{array}{l}\text { This is an Open Access article licensed under the terms of the } \\
\text { Creative Commons Attribution-NonCommercial 3.0 Un- } \\
\text { ported license (CC BY-NC) (www.karger.com/OA-license), } \\
\text { applicable to the online version of the article only. Distribu- } \\
\text { tion permitted for non-commercial purposes only. }\end{array}$
\end{tabular}

trols. HRR indices were similar in the limited and diffuse SSC subgroups. Conclusions: The patients with SSc had lower HRR indices than normal subjects. Cardiac autonomic functions might be involved in SSc, even in patients without cardiac symptoms.

(c) 2014 S. Karger AG, Basel

\section{Introduction}

Systemic sclerosis (SSc) is a systemic, inflammatory and autoimmune disease characterized by immune activation, vasculopathy and fibrosis that is highly heterogeneous in its multisystem clinical manifestations and follows a variable and unpredictable course [1]. Whether presenting in the limited or diffuse form, the progressive fibrotic process, which is a pathologic hallmark of the disease, tends to involve the skin and multiple organ systems, such as the gastrointestinal tract, kidneys and lungs [2]. The heart is one of the major organs involved in SSc and the presence of cardiac involvement generally portends poor prognosis for the patient. Various cardiovascular

Ugur Nadir Karakulak, MD

Department of Cardiology

Faculty of Medicine, Hacettepe University

TR-06100 Ankara (Turkey)

E-Mail ukarakulak@gmail.com 
complications may occur in the natural clinical course of SSc, including rhythm disturbance, pulmonary hypertension and autonomic nervous system (ANS) dysfunction $[3,4]$. Although cardiac involvement can be demonstrated by using sensitive tools in most SSc patients, it is often underestimated due to the occult nature of the signs and symptoms [5]. When cardiac involvement becomes clinically evident, it is recognized as a poor prognostic factor $[6,7]$ and has been shown to be associated with high mortality $(70 \%)$ at 5 years [8]. Hence, the recognition of cardiac dysfunction in affected individuals is important and may influence the overall favorable prognosis.

Heart rate recovery (HRR) after graded exercise is one of the commonly used techniques that reflects autonomic activity $[9,10]$. Sympathetic activity increases during exercise and diminishes during recovery, meaning previously suppressed parasympathetic activity becomes dominant during recovery and reduces the heart rate (HR). This decline is blunted by decreased myocardial function and reduced exercise capacity. Several studies have shown that an abnormal HRR, defined as a failure of the HR to decrease by 12 beats or more during the first minute after peak exercise, independently predicts increased cardiovascular and all-cause mortality rates $[11,12]$.

ANS functions have been previously assessed in SSc using cardiovascular reflex tests [13], HR variability (HRV) $[14,15]$ and HR turbulence (HRT) [16]. However, HRR in SSc patients has not been evaluated. Furthermore, because HRR can be measured more easily and results are much simpler to interpret than HRV and HRT, the present study aimed to evaluate ANS function using HRR indices in SSc patients compared to control subjects.

\section{Subjects and Methods}

\section{Study Population}

One hundred and sixty patients with SSc diagnosed according to American College of Rheumatology [17] and/or LeRoy's classification criteria [2] were enrolled in this study. All subjects had a complete history taken and physical examination performed. Exclusion criteria were patients with diabetes mellitus, hypertension, dyslipidemia, chronic renal failure, chronic liver disease, neurologic diseases, known coronary artery or structural heart disease, pulmonary hypertension, rhythm abnormalities, chronotropic incompetence, drug use which has effects on the autonomic system (such as antiarrhythmics, tricyclic antidepressants and antipsychotics), and cigarette smoking. Chronotropic incompetence was diagnosed when HR failed to reach $85 \%$ of the age-predicted maximal HR when measured during an incremental exercise test. Spirometry and carbon monoxide diffusing capacity (DLCO) were performed in all patients, and those whose forced vital capacity was $\leq 80 \%$ of predicted and whose DLCO was $\leq 80 \%$ of predicted were also excluded [18]. Based on the exclusion criteria, a total of 35 SSc patients were suitable and were included in the study. Thirty-five volunteers matched for age and sex with no previous history of cardiac disease served as the control group. Both the patients and controls were all white Turks. All patients were categorized in the New York Heart Association class I. Patients were classified as having diffuse or limited SSc according to the extent of cutaneous involvement (diffuse SSc: skin thickening proximal to the elbow and/ or knee, with or without face and neck involvement; limited SSc: skin sclerosis confined to digits and small areas of the head and the neck) [17]. Electrocardiography (ECG), treadmill exercise testing and transthoracic echocardiography were performed in all patients. The study was approved by the institution's ethics committee, and patients gave their informed written consent.

\section{Treadmill Exercise Testing}

Treadmill exercise testing was done in all patients using the modified Bruce protocol. Mason-Likar modification of 12-lead ECG [19] was continuously recorded at a paper speed of $25 \mathrm{~mm} / \mathrm{s}$. All patients achieved an exercise time of more than $6 \mathrm{~min}$, and a maximum HR (MHR) of at least $85 \%$ of the age-predicted MHR response. After achieving their peak workload, all patients spent at least 3 min recovering without a cool-down period. Exercise capacity was measured in metabolic equivalent levels (METs) at peak exercise. HRR indices were calculated by subtracting the first-, second- and third-minute HR from the MHR obtained during stress testing and were designated as HRR1, HRR2 and HRR3, respectively. HR reserve was determined as the change in HR from rest to peak exercise during the exercise test. Impaired HRR was described as a decrease in HR from peak exercise to $1 \mathrm{~min}$ of recovery of $<12$ beats per $\min (\mathrm{bpm})$.

\section{Transthoracic Echocardiography}

Standard echocardiographic imaging was performed in the left lateral decubitus position using a commercially available system (Vingmed System Five; GE Vingmed Ultrasound, Horten, Norway). Images were obtained using a 2.5- to $3.5-\mathrm{MHz}$ transducer in the parasternal and apical views. Left ventricular end-diastolic and end-systolic diameters were determined with M-mode echocardiography under two-dimensional guidance in the parasternal long-axis view, according to the recommendations of the American Society of Echocardiography [20]. Left ventricular ejection fraction was calculated from the apical four-chamber view, according to the modified Simpson's rule [20]. Pulsed-wave Doppler was performed in the apical 4-chamber view to obtain mitral inflow indices to assess left ventricular filling according to the recommendations of the American Society of Echocardiography [21]. Measurements of mitral inflow included the peak early filling (E-wave) and late diastolic filling (A-wave) velocities, the E/A ratio, deceleration time (DT) of early filling velocity, and the isovolumic relaxation time (IVRT), derived by placing the cursor of the continuous-wave Doppler in the left ventricular outflow tract to simultaneously display the end of aortic ejection and the onset of mitral inflow. Pulmonary systolic arterial pressure was estimated using continuous-wave Doppler as the peak regurgitation velocity plus an assumed right atrial pressure in relation to the size and respiratory excursion of the inferior cava vein visualized in the subcostal view [20].
18

Med Princ Pract 2015;24:17-22 DOI: $10.1159 / 000368359$
Karakulak et al. 
Statistical Analysis

Statistical analyses were performed using SPSS for Windows 15 (SPSS Inc., Chicago, Ill., USA). Numerical variables with a normal distribution are presented as the mean \pm standard deviation and numerical variables with a skewed distribution are presented as the median with minimum and maximum range, and categorical variables were presented as percentages. For numerical variables, an independent-sample $t$ test and Mann-Whitney $U$ test were used for intergroup comparisons. $\chi^{2}$ test and Fischer's exact $\chi^{2}$ test were used for comparisons of categorical variables. Two-tailed $p$ values $<0.05$ were considered as significant.

\section{Results}

\section{General Characteristics of the Study Population}

The baseline characteristics of the study population are shown in table 1 . In the SSc group, the mean disease duration was $9.1 \pm 3.8$ years (range $3-23.9$ ). There was no statistically significant difference between the patient and control groups in terms of age, sex, systolic and diastolic blood pressures, resting HR and body mass index. All patients and control-group participants had normal left ventricular ejection fraction (mean $66.5 \pm 5.1$ vs. $67.7 \pm$ $5.9 \%, \mathrm{p}=0.40)$ and pulmonary systolic arterial pressure $(24.0 \pm 5.0$ vs. $23.3 \pm 6.4 \mathrm{~mm} \mathrm{Hg}, \mathrm{p}=0.65)$. Mitral E-wave, A-wave, DT and IVRT were similar in the two groups. Of the 35 SSc patients, diffuse SSc was diagnosed in 19 (54\%) and limited SSc in 16 (46\%). The main clinical, laboratory and serologic data of the SSc patients are shown in table 2; $45.7 \%$ had a digital ulcer and $57.1 \%$ had Raynaud's phenomenon. The mean white blood cell count was $6,280 \pm 2,160$ cells/ $\mu$ l, the median C-reactive protein value was $0.35 \mathrm{mg} / \mathrm{dl}$ (range $0-2.68$ ) and the median erythrocyte sedimentation rate was $15 \mathrm{~mm} / \mathrm{h}$ (range $3-71)$.

\section{Exercise Test Parameters and HRR Indices}

All patients and control subjects had a normal resting 12-lead ECG. All subjects completed the exercise stress test without any complications. No rhythm abnormalities or ischemic changes were observed during the ECG stress test in either group. The duration of the treadmill exercise test $(8.9 \pm 2.3$ vs. $9.0 \pm 2.1 \mathrm{~min})$, peak exercise capacity (11.9 \pm 2.3 vs. $12.0 \pm 2.4 \mathrm{METs}), \mathrm{HR}$ reserve $(66.2 \pm 11.9$ vs. $70.9 \pm 9.5 \mathrm{bpm})$ and MHR $(153.6 \pm 5.8$ vs. $155.1 \pm 6.3$ bpm) were similar in the SSc and control groups (table 3). The mean HRR1 (21.8 \pm 4.4 vs. $27.7 \pm 4.3 \mathrm{bpm}, \mathrm{p}=$ $0.001)$, HRR2 ( $43.8 \pm 6.3$ vs. $47.6 \pm 4.4 \mathrm{bpm}, \mathrm{p}=0.004)$ and HRR3 (58.8 \pm 10.3 vs. $63.6 \pm 7.3 \mathrm{bpm}, \mathrm{p}=0.031)$ values were significantly lower in the SSc group than in the control group (fig. 1).
Table 1. Demographic characteristics, blood pressure and echocardiographic parameters of the groups

\begin{tabular}{lccl}
\hline Variable & $\begin{array}{c}\text { SSc group } \\
(\mathrm{n}=35)\end{array}$ & $\begin{array}{l}\text { Control group } \\
(\mathrm{n}=35)\end{array}$ & $\begin{array}{l}\mathrm{p} \\
\text { value }\end{array}$ \\
\hline Age, years & $45.2 \pm 11.6$ & $43.9 \pm 10.0$ & 0.64 \\
Female/male & $31 / 4$ & $31 / 4$ & 1.00 \\
Body mass index & $26.5 \pm 3.2$ & $25.7 \pm 3.0$ & 0.44 \\
Resting heart rate, bpm & $87.4 \pm 12.5$ & $84.2 \pm 9.8$ & 0.25 \\
SBP, mm Hg & $109.6 \pm 12.1$ & $110.4 \pm 12.6$ & 0.77 \\
DBP, mm Hg & $68.4 \pm 8.9$ & $69.1 \pm 9.8$ & 0.31 \\
LVEF, \% & $66.5 \pm 5.1$ & $67.7 \pm 5.9$ & 0.40 \\
SPAP, mm Hg & $24.0 \pm 5.0$ & $23.3 \pm 6.4$ & 0.65 \\
E-wave, cm/s & $77 \pm 16$ & $74 \pm 15$ & 0.50 \\
A-wave, cm/s & $69 \pm 11$ & $68 \pm 10$ & 0.82 \\
Mitral E/A & $1.18 \pm 0.38$ & $1.13 \pm 0.27$ & 0.57 \\
DT, ms & $189.6 \pm 43.5$ & $190.1 \pm 47.0$ & 0.62 \\
IVRT, ms & $98.8 \pm 13.8$ & $97.6 \pm 15.5$ & 0.57 \\
\hline
\end{tabular}

$\mathrm{DBP}=$ Diastolic blood pressure at baseline; $\mathrm{SBP}=$ systolic blood pressure at baseline; $\mathrm{LVEF}=$ left ventricular ejection fraction; SPAP = systolic pulmonary arterial blood pressure.

Table 2. Main clinical, laboratory and serologic data of the SSc patients

Digital ulcers

Raynaud's phenomenon

Hemoglobin, g/dl

White blood cell count, cells/ $\mu \mathrm{l}$

C-reactive protein, $\mathrm{mg} / \mathrm{dl}$

Erythrocyte sedimentation rate, $\mathrm{mm} / \mathrm{h}$

Anti-nuclear antibody positivity

Anti-double-stranded DNA antibodies

Anti-centromere antibodies

Anti-extractable nuclear antigen antibodies

Anti-topoisomerase I (anti-Scl-70) antibodies

$16(45.7)$

$20(57.1)$

$12.5 \pm 1.2$

$6,280 \pm 2,160$

$0.35(0-2.68)$

$15(3-71)$

$23(65.7)$

$4(11.4)$

$8(22.9)$

$21(60)$

$16(45.7)$

$2(5.7)$

Anti-SSA/Ro antibodies

Anti-SSB/La antibodies

0

Anti-Smith antibodies

0

Anti-ribonucleoprotein antibodies

Anti-Jo-1 antibodies

$3(8.6)$

0

Anti-cardiolipin IgM antibodies

$1(2.9)$

Anti-cardiolipin IgG antibodies

$2(5.7)$

Anti-phospholipid IgM antibodies

Anti-phospholipid IgG antibodies

$1(2.9)$

Data are presented as $\mathrm{n}$ with percentage in parentheses, mean \pm standard deviation, or median with min.-max. range in parentheses. 


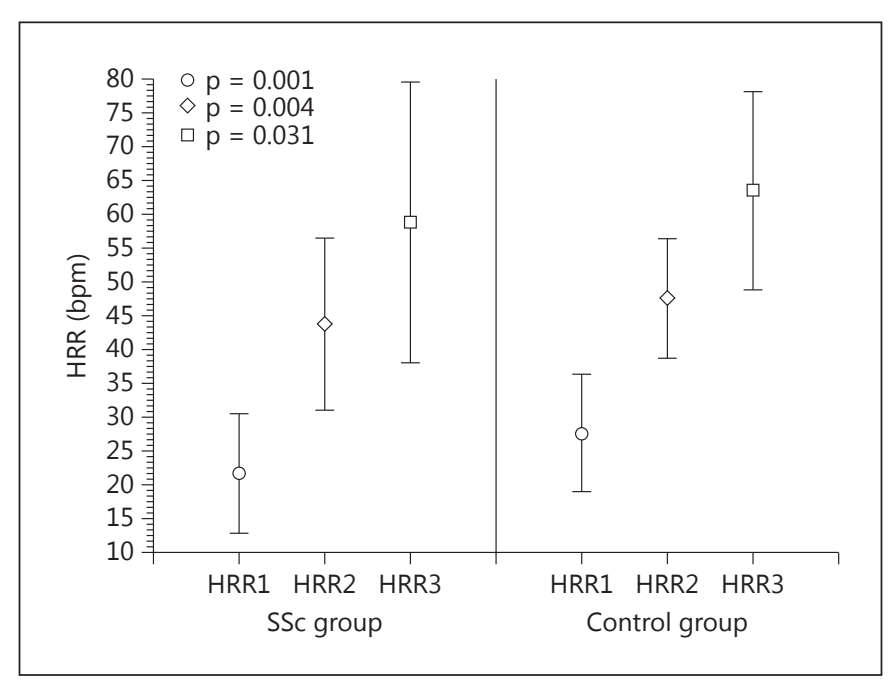

Fig. 1. Comparison of HRR in the SSc and control groups.

Table 3. Exercise test parameters and HRR indices of the groups

\begin{tabular}{lccl}
\hline Variable & $\begin{array}{c}\text { SSc group } \\
(\mathrm{n}=35)\end{array}$ & $\begin{array}{c}\text { Control group } \\
(\mathrm{n}=35)\end{array}$ & $\begin{array}{l}\mathrm{p} \\
\text { value }\end{array}$ \\
\hline Duration of exercise test, min & $8.9 \pm 2.3$ & $9.0 \pm 2.1$ & 0.19 \\
MHR, bpm & $153.6 \pm 5.8$ & $155.1 \pm 6.3$ & 0.30 \\
HR reserve, bpm & $66.2 \pm 11.9$ & $70.9 \pm 9.5$ & 0.07 \\
Maximal SBP, mm Hg & $163.1 \pm 18.7$ & $165.3 \pm 19.2$ & 0.63 \\
Maximal DBP, mm Hg & $85.6 \pm 12.9$ & $86.9 \pm 13.1$ & 0.68 \\
Peak exercise capacity, METs & $11.9 \pm 2.3$ & $12.0 \pm 2.4$ & 0.18 \\
HRR1, bpm & $21.8 \pm 4.4$ & $27.7 \pm 4.3$ & 0.001 \\
HRR2, bpm & $43.8 \pm 6.3$ & $47.6 \pm 4.4$ & 0.004 \\
HRR3, bpm & $58.8 \pm 10.3$ & $63.6 \pm 7.3$ & 0.031 \\
\hline
\end{tabular}

Values represent mean \pm standard deviation. $\mathrm{DBP}=$ Diastolic blood pressure; SBP = systolic blood pressure.

The comparison of the exercise test parameters and HRR indices between the limited SSc and diffuse SSc subgroups revealed that the duration of exercise test, peak exercise capacity and MHR were similar (table 4). The mean HRR1, HRR2 and HRR3 values were also similar in both these subgroups (fig. 2).

\section{Discussion}

The main finding of the present study is that HRR in the first, second and third minute of the recovery period after maximal exercise testing was lower in patients with

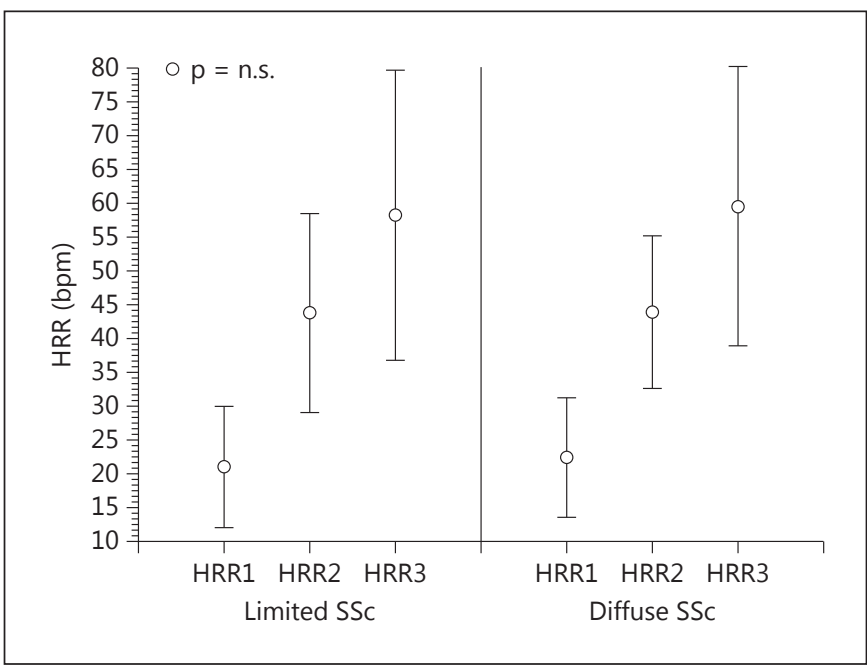

Fig. 2. Comparison of HRR in the limited and diffuse SSc subgroups. n.s. = Not significant.

Table 4. Exercise test parameters and HRR indices between limited and diffuse SSc

\begin{tabular}{lccc}
\hline Variable & $\begin{array}{l}\text { Limited SSc } \\
\text { group }(\mathrm{n}=16)\end{array}$ & $\begin{array}{l}\text { Diffuse SSc } \\
\text { group }(\mathrm{n}=19)\end{array}$ & $\begin{array}{l}\mathrm{p} \\
\text { value }\end{array}$ \\
\hline Duration of exercise test, min & $8.8 \pm 2.4$ & $9.0 \pm 2.1$ & 0.26 \\
MHR, bpm & $154.1 \pm 5.5$ & $153.1 \pm 5.8$ & 0.61 \\
Maximal SBP, mm Hg & $163.7 \pm 16.5$ & $162.5 \pm 13.2$ & 0.74 \\
Maximal DBP, mm Hg & $81.2 \pm 8.3$ & $84.0 \pm 8.7$ & 0.17 \\
Peak exercise capacity, METs & $11.8 \pm 2.3$ & $11.9 \pm 2.4$ & 0.90 \\
HRR1, bpm & $21.1 \pm 4.5$ & $22.4 \pm 4.4$ & 0.37 \\
HRR2, bpm & $43.7 \pm 7.3$ & $43.9 \pm 5.6$ & 0.95 \\
HRR3, bpm & $58.1 \pm 10.7$ & $59.4 \pm 10.3$ & 0.73
\end{tabular}

Values represent mean \pm standard deviation. $\mathrm{DBP}=$ Diastolic blood pressure; SBP = systolic blood pressure.

SSc than in healthy controls, and HRR indices were similar between the limited and diffuse SSc subgroups. Exercise is associated with increased sympathetic and decreased parasympathetic activity. The period of recovery after exercise is accompanied by further dynamic changes in autonomic tone which are clinically characterized by the gradual return of the HR to its resting level. Recovery of the parasympathetic system dominance plays a major role in this gradual return. HRR after graded exercise is one of the commonly used techniques that reflects autonomic activity $[9,22]$. An attenuated HRR, which is defined as a reduction in the decrease in HR immediately after exercise, reflects reduced parasympathetic nervous 
system activity $[23,24]$. Upon cessation of exercise, augmentation of the parasympathetic effects on HR occurs rapidly within the first minute $[22,25]$. In the later stages of recovery, the reduction in sympathetic system regression and dominance of the parasympathetic system causes a decrease in further reduction in HR. This could be the reason why HRR1 was the most statistically significant parameter in our study. During the first minute of recovery, an HR decrease of greater than $20 \mathrm{bpm}$ was found in both groups and this rate of decrease reduced during the second and third minutes of recovery. Moreover, the significance level of HRR2 and HRR3 also decreased compared to HRR1. The significance of HRR1, which was affected more dominantly by the parasympathetic system, between the SSc and the control groups can be considered as another marker representing autonomic dysfunction in the SSc group.

The findings of our study are consistent with a previous study that researched ANS involvement in rheumatologic, autoimmune or inflammatory diseases and its reflection on the cardiac system [26]. Furthermore, the finding that there was no difference in limited and diffuse SSc HRR was consistent with other studies that used HRT [16], HRV and QT dynamic parameters [27]. The lack of a significant difference between diffuse and limited subgroups could be due to worsening of the HRR index independent of the degree of involvement and, thus, may be an indicator of early-phase cardiac involvement. Klimiuk et al. [13], using the Valsalva maneuver, reported a decreased ratio of the longest $\mathrm{R}-\mathrm{R}$ interval to the shortest $\mathrm{R}-\mathrm{R}$ interval in SSc patients compared to healthy individuals. Ferri et al. [15], who performed HRV analysis during 24-hour ambulatory ECG monitoring in 30 adult patients with SSc, reported that time-domain and spectral-domain parameters were significantly lower in SSc patients.

Our finding that none of the patients had significant pulmonary function abnormalities detected by spirometry contrasts with the study of Pancera et al. [28]. They observed a negative correlation between HRV and respiratory parameters in SSc subjects, suggesting that impairment of respiratory function could be a cause of alteration in autonomic control and normal pulmonary structure required for the autonomic modulation of cardiac function. In our study, a probable explanation could be that blunted HRR indices might be an early sign of ANS involvement in SSc, prior to the emergence of respiratory abnormalities.

In our study, although peak exercise capacity and MHR were similar in the two groups, HRR indices were

lower in patients with SSc, possibly indicating the presence and early blunting of autonomic dysfunction in SSc. Exercise capacity in patients with SSc is reduced due to pulmonary fibrosis, pulmonary hypertension and muscular involvement. In our institution, the clinical course of these patients is followed with the 6-min walking test. However, it has been shown that exercise capacity can decrease even without pulmonary involvement [29].

The limitations of this study include the fact that it was based on cross-sectional data, the number of patients included was small and there was no information regarding any cardiovascular end points. Because of the limited number of patients due to several exclusion criteria, the findings of this study should not be generalized to all SSc patients. Larger studies with follow-up of cardiovascular end points are needed to better understand the pathophysiology of SSc and to enable accurate clinical decisions to be made.

\section{Conclusion}

SSc was associated with a delayed recovery of HR after graded maximal exercise. The prognostic significance of HRR in patients with SSc should be followed closely for adverse cardiovascular outcomes. Further studies are required to understand the pathogenesis and consequences of autonomic dysfunction in SSc.

References

Med Princ Pract 2015;24:17-22 DOI: $10.1159 / 000368359$
${ }_{1}$ Jimenez SA, Derk CT: Following the molecular pathways toward an understanding of the pathogenesis of systemic sclerosis. Ann Intern Med 2004; 140:37-50.

2 LeRoy EC, Black C, Fleischmajer R: Scleroderma (systemic sclerosis): classification, subsets and pathogenesis. J Rheumatol 1988;15: 202-205.

- 3 Allanore Y, Meune C, Kahan A: Outcome measures for heart involvement in systemic sclerosis. Rheumatology 2008;47(suppl 5): v51-v53.

4 Seferovic PM, Ristic AD, Maksimovic R: Cardiac arrhythmias and conduction disturbances in autoimmune rheumatic diseases. Rheumatology 2006;45(suppl 4):39-42.

5 Vachiery JL, Coghlan G: Screening for pulmonary arterial hypertension in systemic sclerosis. Eur Respir Rev 2009;18:162-169.

-6 Kepez A, Akdogan A, Sade LE, et al: Detection of subclinical cardiac involvement in systemic sclerosis by echocardiographic strain imaging. Echocardiography 2008;25:191-197. 
7 Kahan A, Allanore Y: Primary myocardial involvement in systemic sclerosis. Rheumatology 2006;45(suppl 4):14-17.

8 Medsger TA Jr, Masi AT: Survival with scleroderma. II. A life-table analysis of clinical and demographic factors in 358 male US veteran patients. J Chronic Dis 1973;26:647-660.

-9 Okutucu S, Kabakci G, Deveci OS, et al: Relationship between exercise heart rate recovery and circadian blood pressure pattern. J Clin Hypertens 2010;12:407-413.

10 Gibbons RJ: Abnormal heart-rate recovery after exercise. Lancet 2002;359:1536-1537.

-11 Morshedi-Meibodi A, Larson MG, Levy D, et al: Heart rate recovery after treadmill exercise testing and risk of cardiovascular disease events (the Framingham heart study). Am J Cardiol 2002;90:848-852.

12 Cole CR, Blackstone EH, Pashkow FJ, et al: Heart-rate recovery immediately after exercise as a predictor of mortality. N Engl J Med 1999;341:1351-1357.

13 Klimiuk PS, Taylor L, Baker RD, et al: Autonomic neuropathy in systemic sclerosis. Ann Rheum Dis 1988;47:542-545.

14 Morelli S, Piccirillo G, Fimognari F, et al: Twenty-four hour heart period variability in systemic sclerosis. J Rheumatol 1996;23:643645.

15 Ferri C, Emdin M, Giuggioli D, et al: Autonomic dysfunction in systemic sclerosis: time and frequency domain 24 hour heart rate variability analysis. Br J Rheumatol 1997;36: 669-676.
16 Bienias P, Ciurzynski M, Glinska-Wielochowska $\mathrm{M}$, et al: Heart rate turbulence assessment in systemic sclerosis: the role for the detection of cardiac autonomic nervous system dysfunction. Rheumatology 2010;49: 355-360.

$>17$ Subcommittee for Scleroderma Criteria of the American Rheumatism Association Diagnostic and Therapeutic Criteria Committee: Preliminary criteria for the classification of systemic sclerosis (scleroderma). Arthritis Rheum 1980;23:581-590.

18 Furst DE: Rational therapy in the treatment of systemic sclerosis. Curr Opin Rheumatol 2000; $12: 540-544$

19 Papouchado M, Walker PR, James MA, et al: Fundamental differences between the standard 12-lead electrocardiograph and the modified (Mason-Likar) exercise lead system. Eur Heart J 1987;8:725-733.

20 Lang RM, Bierig M, Devereux RB, et al: Recommendations for chamber quantification: a report from the American Society of Echocardiography's Guidelines and Standards Committee and the Chamber Quantification Writing Group, developed in conjunction with the European Association of Echocardiography, a branch of the European Society of Cardiology. J Am Soc Echocardiogr 2005;18:14401463.

21 Nagueh SF, Appleton CP, Gillebert TC, et al: Recommendations for the evaluation of left ventricular diastolic function by echocardiography. J Am Soc Echocardiogr 2009;22:107133.
22 Kaya EB, Okutucu S, Aksoy H, et al: Evaluation of cardiac autonomic functions in patients with ankylosing spondylitis via heart rate recovery and heart rate variability. Clin Res Cardiol 2010;99:803-808.

23 Vivekananthan DP, Blackstone EH, Pothier $\mathrm{CE}$, et al: Heart rate recovery after exercise is a predictor of mortality, independent of the angiographic severity of coronary disease. J Am Coll Cardiol 2003;42:831-838.

24 Arena R, Guazzi M, Myers J, et al: Prognostic value of heart rate recovery in patients with heart failure. Am Heart J 2006;151:851.e7e13.

25 Okutucu S, Oto A: Risk stratification in nonischemic dilated cardiomyopathy: current perspectives. Cardiol J 2010;17:219-229.

26 Sarli B, Dogan Y, Baktir AO, et al: Heart rate recovery is impaired in patients with psoriasis. Med Princ Pract 2013;22:567-570.

27 Ciftci O, Onat AM, Yavuz B, et al: Cardiac repolarization abnormalities and increased sympathetic activity in scleroderma. J Natl Med Assoc 2007;99:232-237.

28 Pancera P, Sansone S, Presciuttini B, et al: Autonomic nervous system dysfunction in sclerodermic and primary Raynaud's phenomenon. Clin Sci 1999;96:49-57.

29 de Oliveira NC, dos Santos Sabbag LM, Ueno LM, et al: Reduced exercise capacity in systemic sclerosis patients without pulmonary involvement. Scand J Rheumatol 2007;36: 458-461. 